\title{
Computing spatial distribution of tube and louvre luminaires efficiency from their description
}

\author{
Jean-Marc DENIEL ${ }^{1, *}$ \\ ${ }^{1}$ INRS, Institut national de recherche et de sécurité, Nancy, FRANCE
}

Received 05 December 2019, Accepted 09 June 2020

\begin{abstract}
Lighting computation requires photometry data that are not always available. Lacking photometry data limits lighting study to in situ measurement, luminaire measurement or use of similar luminaire photometry. This is not satisfactory, neither for convenience nor cost and accuracy reasons. Fitting the spatial distribution of luminaire efficiency to their description would allow lighting computations in this kind of situation. An efficiency spatial distribution model is proposed for grid and louvre tube luminaires, taking optic width, louvre between-axis and gloss as parameters. It is constructed over 12 measured efficiency spatial distributions and the corresponding luminaire descriptors. Even if optic and louvre gloss cannot be differentiated, this model fits to measurements and allows for computed irradiance close to experiments within $-5 \%$ to $+19 \%$. In addition, luminaire descriptors can freely vary inside their experimental range and even be extrapolated.
\end{abstract}

Keywords: photometry, light sources, luminaires, lighting, simulation, model

\section{Introduction}

Lighting simulation is a tool for appraising lighting quality in indoor environments. Such software [1,2] is usually based on global illumination techniques [3]. This technique takes light sources into account through lamp luminous power and luminaire photometry [4], both being usually given by manufacturers.

Lighting designers have to face two kinds of situation. On the one hand, the lighting installation will be new. Then, luminaires photometry will be available in software databases.

On the other hand, an existing installation is to be appraised. For example:

- this used installation, once relamped or retrofitted with LED tubes, may be compared with a brand new one

- the studied area is modified or re-organized while keeping as much as possible of the existing.

In such cases, lighting designers may miss the photometry data of the luminaires for various reasons. For instance, the manufacturer did not publish them (which is the case of low-grade or custom-made luminaires). Another reason is that old luminaires removed from catalogues tend to disappear from software databases.

In such a situation, lighting can be assessed and rendered using one of these alternative methods:

- the lighting designer can use the photometry datafile of another light source. It supposes that the two photometries can be compared (at least on diagrams)

- the light source has to be unmounted and send to a laboratory for its photometry to be measured [4]. Even if feasible, it is very inconvenient, time-consuming and expensive

- the luminaire efficiency spatial distribution can be computed. It was a research theme using finite-element methods [5], ray-tracing [6,7] and global illumination [8]. While accurate, this way requires finely modeling the shape of all the reflector parts and measuring their bidirectional reflectance distribution function. All this is difficult and time-consuming: its money and time costs are unlikely to be acceptable

- illuminance can be measured in-situ. This does not permit to assess the effects of modifying the lighted area (ground/wall/ceiling materials, furniture, space partitioning). In addition, it does not allow any comparison with alternate lighting proposals since used lamps were measured.

* J.M. DENIEL, tel +33-3-83-50-85-24,

E-mail address: jean-marc.deniel@inrs.fr 
This paper proposes a more convenient solution: predicting the spatial distribution of the efficiency of a luminaire from its description (usually expressed as $\mathrm{cd} / \mathrm{klm}$ ). To ease this paper readability, the "efficiency" term will stand for "spatial distribution of efficiency".

Indoor light sources usually consist in round or parabolic spotlights with discharge or halogen lamps, or grid and louvre luminaires with tubes. This paper addresses the problem of furnishing the photometry for any described louvre luminaire with tubes, which is the most common configuration in workplaces. To our knowledge, this has not been done before.

Our problem is to deduce the efficiency of a louvre luminaire from its description. Modelling efficiency values as already been done, in order to save memory and computation time, using neural networks [9,10]. These papers show that a small perceptron neural network (less than 26 neurons) is sufficient to represent a real-case efficiency. This indicates that an efficiency shows characteristics that allows its modelling.

We have to go a step further: modelling efficiencies from luminaire descriptions. It means proposing a coherent relationship between luminaire description and efficiency characteristics.

These descriptions will be based on optics proportions and a visual appraisal of optics gloss (ranging from 0: matt, to 1: mirror polish). In addition, several hypotheses and restrictions need to be made:

- the proposed work covers light sources that emit their luminous power through one virtual plane. Such an "area light source" is considered as a planar rectangle

- this emitting rectangle is evenly cut into rectangular patches. Each patch covers one optic width and louvres between-axes (see figure 1) as proposed in [11]

- patches can be of two types: whether they are placed along tubes or correspond to a tube end

- anywhere on the emitting plane and for every direction of emission, luminaire efficiency is considered independent from wavelengths between $380 \mathrm{~nm}$ and $780 \mathrm{~nm}$. This means that optics reflectance is "uncoloured"

- anywhere on a given patch, efficiency is the same

- all patches of the same patch type share the same efficiency.

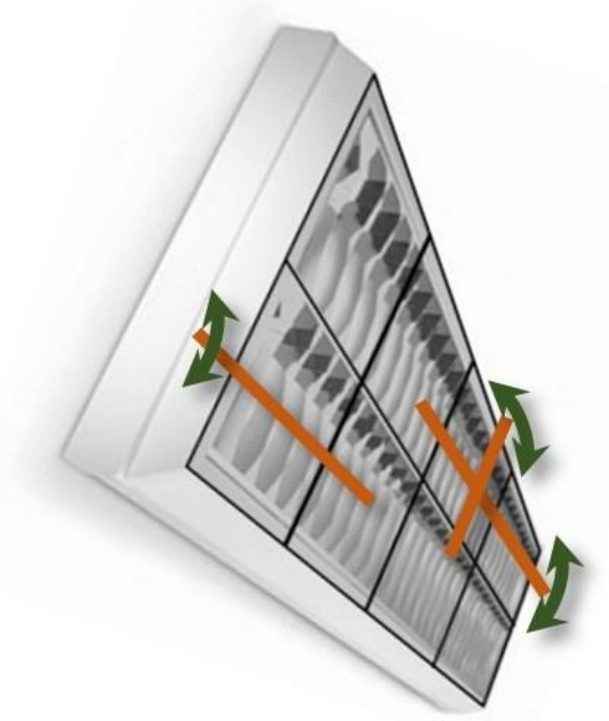

Figure 1: Louvre luminaires are considered as plane rectangular light sources cut into patches. The green arrows show the symmetry axis - showed in brown - for each patch type - at tube end or along tube body. For readability issues, patches showed here as black frames cover several louvres between-axes (the visual dissymmetry in the luminaire photography is due to reflection on the glossy louvers).

Illumination is usually computed by first spreading light from primary light sources: sky through windows and artificial lighting. Artificial light sources are modelled whether as a point source or an area source. Associated units are respectively radiant intensity $(\mathrm{cd}=\mathrm{lm} / \mathrm{sr})$ and luminance $(\mathrm{cd} / \mathrm{m} 2)$. Both units account for lamp luminous power (lm) and luminaire efficiency (cd/klm in photometry datasheets).

Among available photometry coordinate systems, this paper will use photometry $(\mathrm{C}, \gamma)$ coordinates along CIE Cplanes [12] (see figure 2) equivalent to IES $(\theta, \psi)$ type C photometry [4]. 
The proposed model produces efficiencies in the far-field form [4] to comply with illumination software, as opposed to near-field photometry [13].

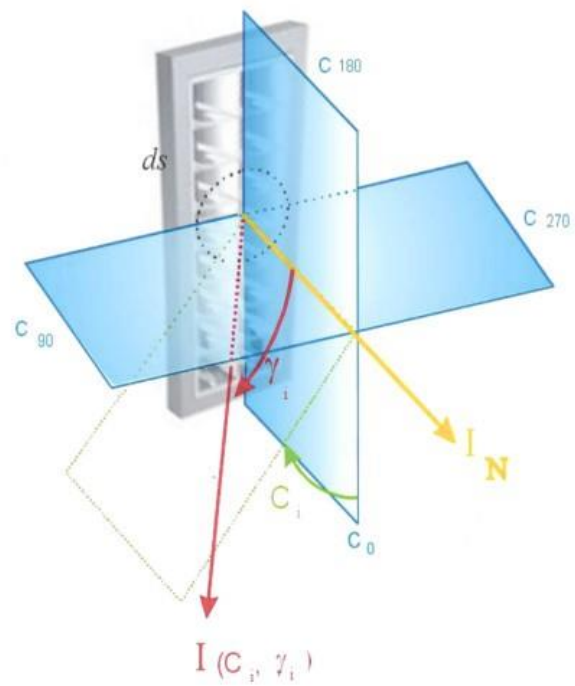

Figure 2: (C, $\gamma)$ CIE C-planes photometry coordinate system.

\section{Materials and method}

\subsection{Samples}

The materials consisted in louvre luminaires described by:

- their optic gloss appraisal and their louvres gloss appraisal, ranging from 0 (matt) to 1 (mirror polish)

- their optic width, that is the width of a single tube reflector. In figure 3, the four-tube luminaires dimensions are $600 \times 600 \mathrm{~mm}$ so an optic width is roughly $140 \mathrm{~mm}$.

- their louvres between-axis.

To be independent from luminaire scale, $\frac{\text { optic width }}{\text { louvre between_axis }}$ ratio was considered. Four luminaires were used. Louvres could be removed (see figure 3), thus giving 12 samples so that $\frac{\text { optic width }}{\text { louvre between_axis }}$ ranges from 0.25 to 2.36 . 

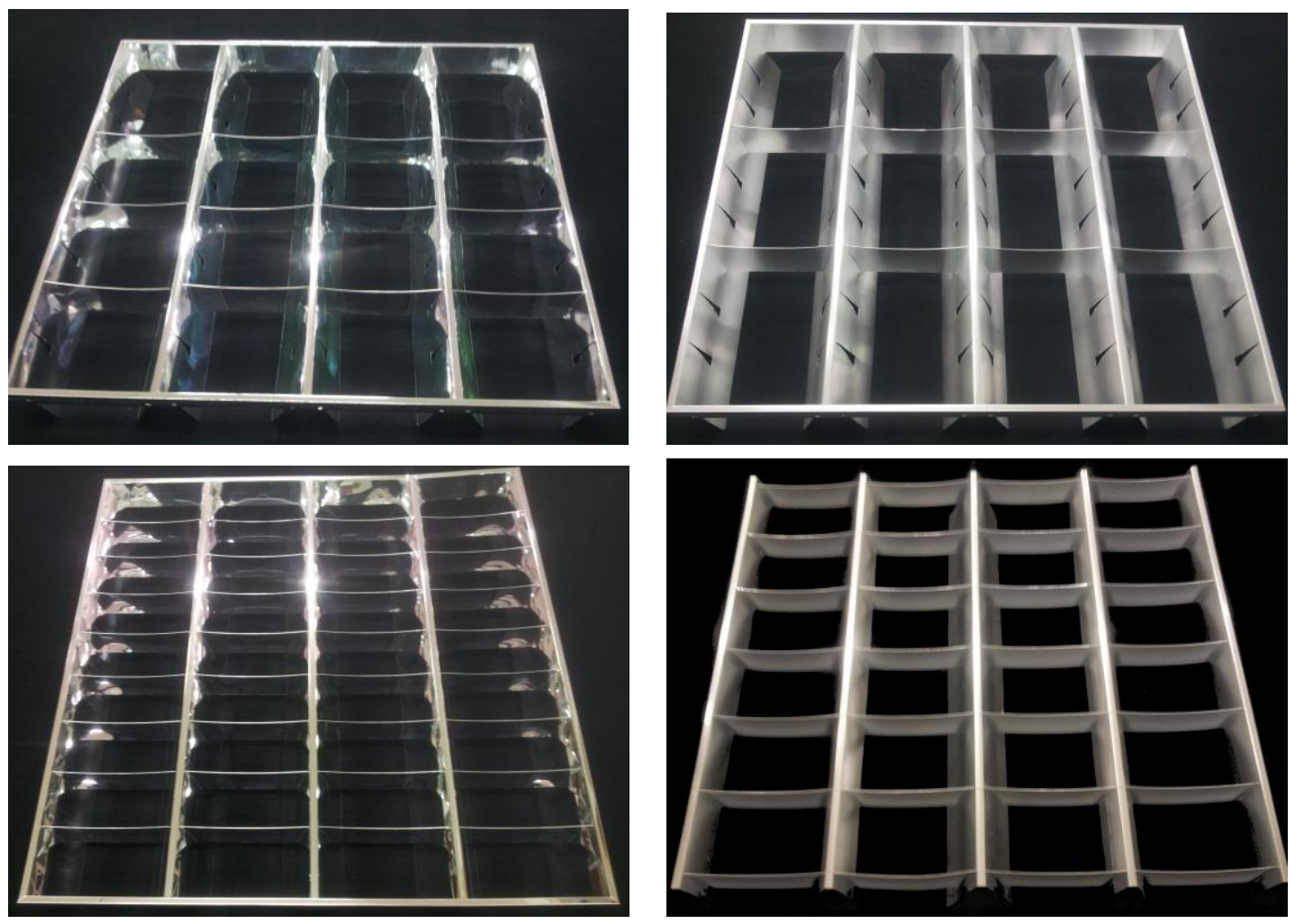

Figure 3: The four sample luminaires used in this paper with some louvre removed. Top-left: One louvre was removed every 2. Top-right: Two louvres were removed every 3 . Bottom: No louvre was removed.

\subsection{Measurement devices}

As exposed before, two patch types exist in louvre luminaires. We had to measure the individual efficiency of each patch in each luminaire configuration, hence using near-field photometry.

In a dark room stabilized in power and temperature, two devices were used in order to retrieve patches efficiency. First, a double monochromator spectroradiometer was used to get an absolute illuminance value at a given distance from the luminaire, facing the centre of its emitting rectangle. Then a near-field photometry spectro-videogoniophotometer was employed in order to obtain the relative distribution of the luminaire efficiency for each patch and confirm the uncoloured-photometry hypothesis. At last raw data were processed in order to be modelizable.

\subsubsection{Use of the spectroradiometer}

A calibrated double monochromator OL750 from Gooch and Housego was used in order to retrieve spectral irradiance values $\mathrm{Ee}, \mathrm{OL} 750(\lambda)$ in front of the measured luminaire at a dOL750 distance between the centre of the luminaire emitting rectangle and the entry of the OL750 integrating sphere. $\lambda$ ranged from $380 \mathrm{~nm}$ to $780 \mathrm{~nm}$.

An EV,OL750 illuminance value is computed from Ee,OL750( $\lambda$ ), and irradiance from $380 \mathrm{~nm}$ to $780 \mathrm{~nm}$ too.

At last, to avoid biasing the measurements because of the tubes orientation, the tubes were set horizontally.

\subsubsection{Use of the near-field spectro-video-goniophotometer}

The scheme of a goniophotometer is given in figure 4, as originally described in [13]. This apparatus is composed of:

- a rotating arm and a rotating and vertically moving platform in order to get sensors to be moved all around the hemisphere facing the luminaire emitting plane turned upward.

- sensors set at the head of the arm, turned toward the arm rotation axis: camera, CCD spectroradiometer and luxmeter. 


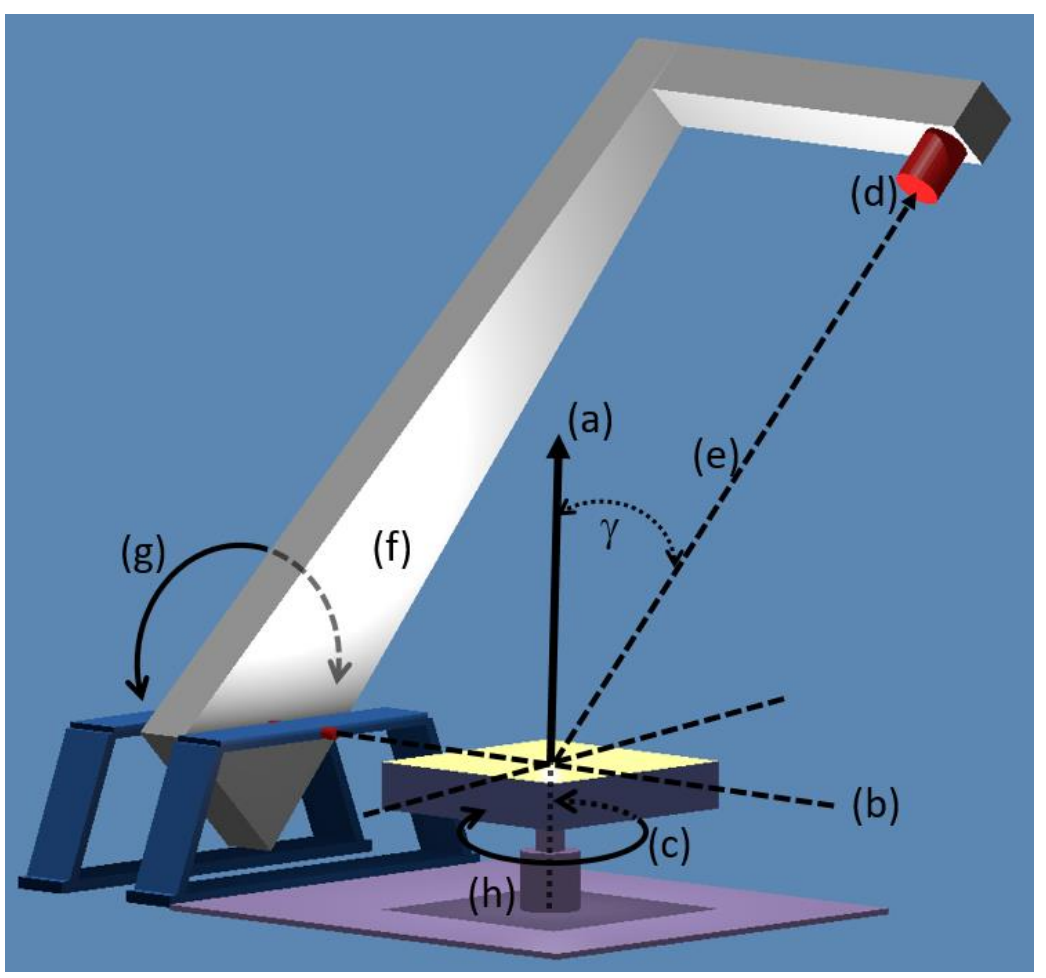

Figure 4: Schema of a photogoniometer. Sensors (d, in red) are installed at the head of an arm (f) and can describe a semi-circle (g) around a light source. This source is turned upward (a) and fixed on a rotating plate (h) so that sensors can describe a hemisphere around it. The light source emitting plane (in yellow) is lifted up to the level of the arm turning axis (b). Then the (g) rotation of the arm corresponds to the $\gamma$ coordinate and the (c) rotation of the source corresponds to the $\mathrm{C}$ angle.

In the following, we note $\left(\begin{array}{l}C \\ \gamma\end{array}\right)$ the angular coordinates of the direction from the center of the emitting rectangle toward the arm head. In this case, the platform is turned to $C$ angle and the arm is inclined to $\gamma$.

The camera is a 16 bits greyscale Sony XCD-SX90 with a neutral density filter and a 25mm lens. Its perspective was corrected by software.

The CCD spectroradiometer is an AvaSpec-2048TEC-USB2 from Avantes. To measure irradiance from $200 \mathrm{~nm}$ to $1,100 \mathrm{~nm}$, it is equipped with a 2,048 pixels linear 16 bits CCD array cooled by Peltier effect.

The luxmeter, with remote head is from Alborn.

\subsection{Measurement protocol}

Measuring luminaires was split into three stages to get efficiencies that can be modelled.

\subsubsection{Preliminary stage}

Camera picture and illuminance and spectral irradiance[14] were measured every $90^{\circ}$ along $C$ (from $0 \circ$ to $270^{\circ}$ included) and every $45^{\circ}$ along $\gamma$ (from $0 \circ$ to $90^{\circ}$ included). These data were only used to confirm the "uncoloured reflectance" of luminaires hypothesis.

\subsubsection{Measuring stage}

During the measuring stage, arm head $\left(\begin{array}{l}\mathrm{C} \\ \gamma\end{array}\right)$ directions covered the hemisphere facing the luminaire emitting plane. Angular resolution was $4 \circ$ at best. The goal of this main stage was, for each $\left(\begin{array}{l}\mathrm{C} \\ \gamma\end{array}\right)$ arm head position and for each $\mathrm{k}$ patch composing the luminaire emitting rectangle, to approach its luminance 
$\mathrm{L}_{\mathrm{V}, \mathrm{k}}\left(\begin{array}{l}\mathrm{C} \\ \gamma\end{array}\right)$.

Only the luxmeter and camera were used. Illuminance $\mathrm{E}_{\mathrm{V}}\left(\begin{array}{l}\mathrm{C} \\ \gamma\end{array}\right)$ was measured and a picture from the camera was retrieved and analysed to compute, for each $\mathrm{k}$ patch composing the emitting rectangle, the average pixel level $\mathrm{L}_{\mathrm{Cam}, \mathrm{k}}\left(\begin{array}{l}\mathrm{C}_{\mathrm{k}} \\ \gamma_{\mathrm{k}}\end{array}\right)$ along the $\left(\begin{array}{l}\mathrm{C}_{\mathrm{k}} \\ \gamma_{\mathrm{k}}\end{array}\right)$ direction from the centre of the patch toward the arm head. Then $\mathrm{L}_{\mathrm{V}, \mathrm{k}}\left(\begin{array}{l}\mathrm{C}_{\mathrm{k}} \\ \gamma_{\mathrm{k}}\end{array}\right)$ was approximated following equation (1).

$$
L_{V, k}\left(\begin{array}{c}
C_{k} \\
\gamma_{k}
\end{array}\right) \approx \frac{E_{V}\left(\begin{array}{c}
C \\
\gamma
\end{array}\right)^{\text {Corr }} L_{C a m, k}\left(\begin{array}{c}
C_{k} \\
\gamma_{k}
\end{array}\right)}{\sum_{k^{\prime}} L_{C a m, k^{\prime}}\left(\begin{array}{c}
C_{k^{\prime}} \\
\gamma_{k^{\prime}}
\end{array}\right) G_{k^{\prime}}\left(\begin{array}{l}
C \\
\gamma
\end{array}\right)}
$$

- where:

$-\left(\begin{array}{l}C_{k} \\ \gamma_{k}\end{array}\right)$ is the direction from the centre of $\mathrm{k}$ toward the arm head

$-k^{\prime}$ stands for all the patches composing the emitting rectangle, $\left(\begin{array}{l}C_{k^{\prime}} \\ \gamma_{k^{\prime}}\end{array}\right)$ being the direction from their center toward the arm head

$-E_{V}\left(\begin{array}{l}C \\ \gamma\end{array}\right)^{\operatorname{Corr}}=\frac{E_{V, O L 750}}{E_{V}\left(\begin{array}{l}0 \\ 0\end{array}\right)} E_{V}\left(\begin{array}{l}C \\ \gamma\end{array}\right)$ is a corrected illuminance value with the arm head in position $\left(\begin{array}{l}C \\ \gamma\end{array}\right)$

$-G_{k^{\prime}}\left(\begin{array}{l}C \\ \gamma\end{array}\right)$ is the projected form factor corresponding to the $k^{\prime}$ patch as seen from the arm head in $\left(\begin{array}{l}C \\ \gamma\end{array}\right)$ position. Its exact expression can be found in $[15]^{1 .}$

\subsubsection{Efficiency extraction stage}

The goal of this third stage was to extract raw photometrical data and to filter them in order to obtain a modelable efficiency.

To be measured, a luminaire was fitted with lamps corresponding to a total luminous flux $\Phi_{V}$ ( $\operatorname{lm}$ ), from manufacturer data.

Let us consider the k patches of the same type. They all share the same $\mathrm{S}(\mathrm{m} 2)$ area. The theoretical relationship between $L_{V, k}\left(\begin{array}{l}C_{k} \\ \gamma_{k}\end{array}\right)$ and the efficiency $\eta\left(\begin{array}{l}C_{k} \\ \gamma_{k}\end{array}\right)$ of this type of patch is given in (2).

$$
L_{V, k}\left(\begin{array}{c}
C_{k} \\
\gamma_{k}
\end{array}\right)=\eta\left(\begin{array}{c}
C_{k} \\
\gamma_{k}
\end{array}\right) \frac{\Phi_{V}}{S}
$$

As previously showed in [16], raw luminance data contain high frequencies variations over $\mathrm{C}$ and $\gamma$ due to noise and caustics. Smoothed efficiency values $\eta\left(\begin{array}{l}C_{k} \\ \gamma_{k}\end{array}\right)$ were obtained for all available $\left(\begin{array}{l}C_{k} \\ \gamma_{k}\end{array}\right)$ directions from equation (1) by:

- averaging on similar directions when accounting for the single and double symmetry axis for tube-end and alongtube-body patch type respectively (see figure 1)

- for each patch type, averaging $\eta\left(\begin{array}{l}C_{k} \\ \gamma_{k}\end{array}\right)$ over all the $\mathrm{k}$ patches of this type

- at last, applying a floating average filter limited to 5 samples or $10^{\circ}$ on $C_{k}$ and $\gamma_{k}$.

It should be noted that a goniometer arm $(\mathrm{C}, \gamma)$ position corresponds to several $\mathrm{C}_{\mathrm{k}}$ and $\gamma_{\mathrm{k}}$ emitting angles from the $\mathrm{k}$ patches toward the arm head. Hence, $C_{k}$ and $\gamma_{k}$ resolution is finer than that of $C$ and $\gamma$.

\subsection{Efficiency data}

Applying the described protocol on above samples, 12 efficiencies were measured. Tube-end and along-tube-body patches efficiencies do not differ much. That's why along-tube-body efficiencies only are considered in the following.

\footnotetext{
1. Erratum: the formula result presented in this thesis should be divided by a factor two.
} 
They are shown in table 1 .

Table 1: Spherical representation of the relative variation of measured efficiency from sample configurations. The $\left(\begin{array}{l}0 \\ 0\end{array}\right)$ normal direction to the emission plane is upward. $\left(\begin{array}{c}180 \\ 90\end{array}\right)$ and $\left(\begin{array}{c}270 \\ 90\end{array}\right)$ directions are indicated too. $\mathrm{P}=\frac{\text { optic width }}{\text { louvre between-axis }}$ and $\mathrm{G}=$ optic gloss are indicated for each sample.

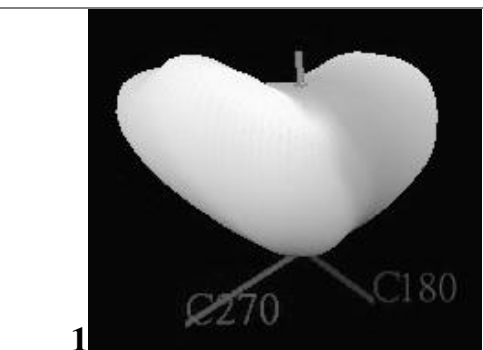

$P=2.33 \mathrm{G}=1$

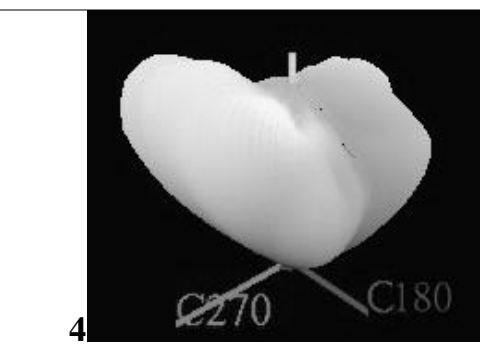

$P=1.17 G=1$

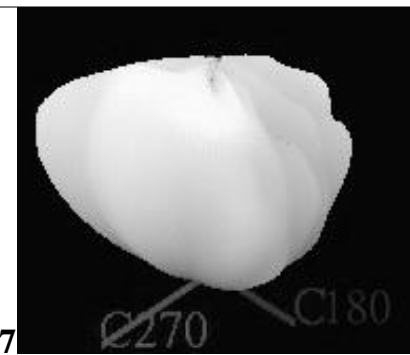

$P=2.37 \quad G=1$

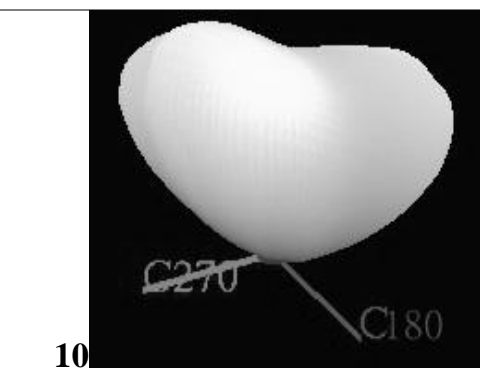

$P=0.59 G=0.5$

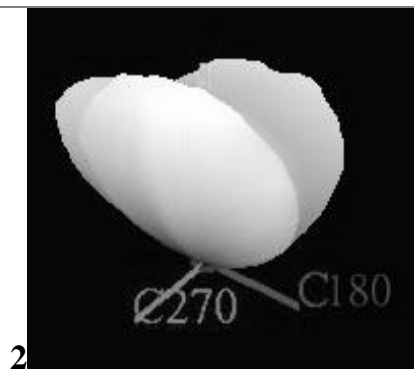

$P=0.25 G=1$

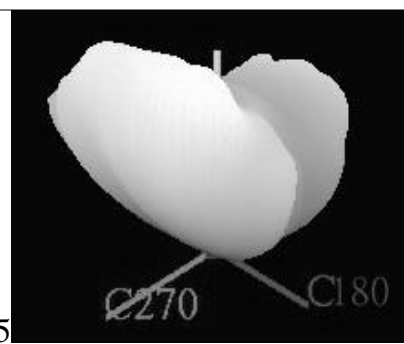

$\mathrm{P}=0.78 \mathrm{G}=1$

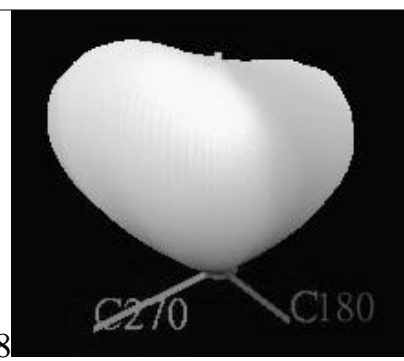

$\mathrm{P}=2.35 \mathrm{G}=0.5$

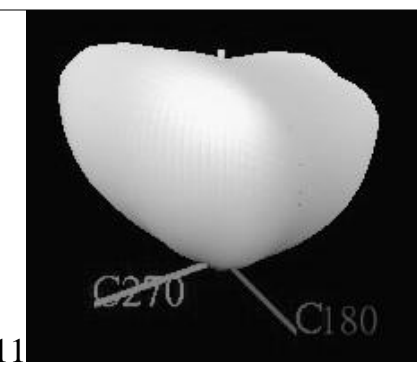

$\mathrm{P}=1.18 \mathrm{G}=0.5$

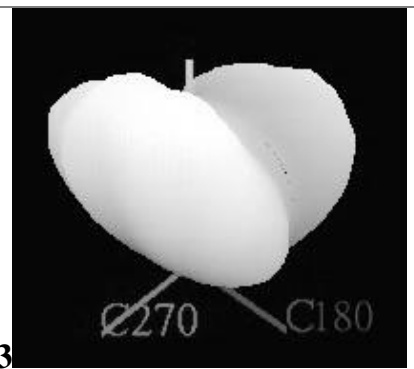

$P=0.58 G=1$

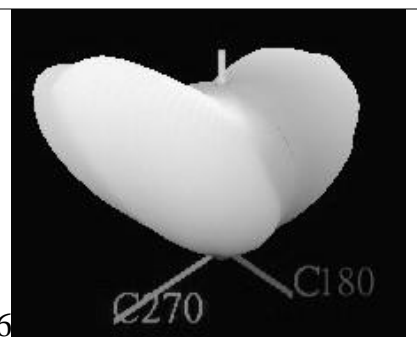

$\mathrm{P}=1.58 \mathrm{G}=0.5$

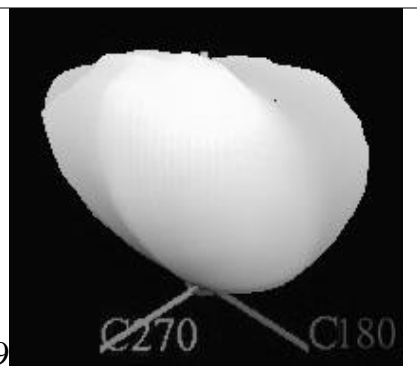

$\mathrm{P}=0.25 \mathrm{G}=0.5$

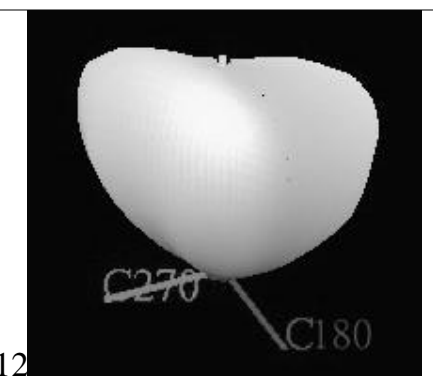

$\mathrm{P}=0.79 \mathrm{G}=0.5$

\subsection{Efficiency model}

Only along-the-tube-body patch type being considered, efficiency to model will simply be notated $\eta\left(\begin{array}{l}\mathrm{C} \\ \gamma\end{array}\right)$. Modelling $\eta\left(\begin{array}{l}C \\ \gamma\end{array}\right)$ consists in expressing it as a function of $\left(\begin{array}{l}C \\ \gamma\end{array}\right)$ and $\frac{\text { optic width }}{\text { louvre between-axis }}$ and optic gloss - ignoring louvre gloss is 
discussed later. The proposed method consists in a $\left(\begin{array}{l}\mathrm{C} \\ \gamma\end{array}\right)$ indexed array of multiple linear regression parameters $\widehat{A}$ such as:

$$
\eta\left(\begin{array}{l}
C \\
\gamma
\end{array}\right) \approx V \cdot \hat{A}
$$

where the row vector $\mathrm{V}$ is $\mathrm{V}=\left(1, \frac{\text { optic width }}{\text { louvre between-axis }}\right.$, optic gloss $)$.

To find $\widehat{A}$ for each $\left(\begin{array}{l}\mathrm{C} \\ \gamma\end{array}\right)$ emitting direction, we note

$-\mathrm{Y}$, a vector of dependent variables. They correspond to $\eta\left(\begin{array}{l}\mathrm{C} \\ \gamma\end{array}\right)$ for each $\mathrm{i}=1$..n experiment $($ here $\mathrm{n}=12)$

$-\mathrm{X}$ is a matrix of $\mathrm{n} \times(\mathrm{p}+1)$ independent variables. In our case, $\mathrm{p}=2$ and for each $\mathrm{i}$ experiment, $\mathrm{Xi}, 0=1, \mathrm{Xi}, 1=$ $\frac{\text { optic width }}{\text { louvre between-axis }}$ and $\mathrm{Xi}, 2$ is optic gloss

$-\mathrm{E}$ is the model error vector

$\hat{A}$ is to be determined according equation (4) to best fit experiments.

$$
\left(\begin{array}{c}
Y_{1} \\
\vdots \\
Y_{n}
\end{array}\right)=\left(\begin{array}{ccc}
1 & X_{1,1} & X_{1,2} \\
\vdots & \vdots & \vdots \\
1 & X_{n, 1} & X_{n, 2}
\end{array}\right)\left(\begin{array}{c}
\hat{A}_{0} \\
\hat{A}_{1} \\
\hat{A}_{2}
\end{array}\right)+\left(\begin{array}{c}
E_{1} \\
\vdots \\
E_{n}
\end{array}\right)
$$

The solution minorizing $E$ is given in equation (5).

$$
\hat{A}=\left(\mathrm{X}^{\mathrm{T}} \mathrm{X}\right)^{-1} \mathrm{X}^{\mathrm{T}} \mathrm{Y}
$$

\section{Results}

Subsequent results account for irradiance, whereas lighting design is based on illuminance. The difference between irradiance and illuminance is taking into account the $\mathrm{V}(\lambda)$ photopic filter. In our case, lamps are identical in simulations and measurement so illuminance ratios would equal irradiance ratios. That is why, in our case, using illuminance is useless and justify the use of irradiance.

\subsection{Confirmed hypotheses}

Spectral irradiance was measured around each sample luminaire. Its relative spectral distribution varies by a few percent along $\mathrm{C}$ and $\gamma$. This makes the optics "uncoloured reflectance" hypothesis acceptable.

Measured luminaires emitting plane was cut into patches. Each patch covered one optic width and one louvre between-axis. Two patch types exist: along tube body and at tube end. The latter is neglected in the proposed model, without noticeably influencing irradiance computation accuracy, as shown in tables 4 and 5.

\subsection{Model limits in terms of gloss}

Optic gloss only differs from louvres in the sixth experiment. Indeed, such configurations are very uncommon among luminaires. Nevertheless, we tested an efficiency model distinguishing optic and louvres gloss. This led to incoherent efficiency in case optic gloss differed from louvres gloss (see figure 5).

That is why, in the $\mu\left(\begin{array}{l}C \\ \gamma\end{array}\right)$ proposed model, louvres and optic are considered as equally glossy. 

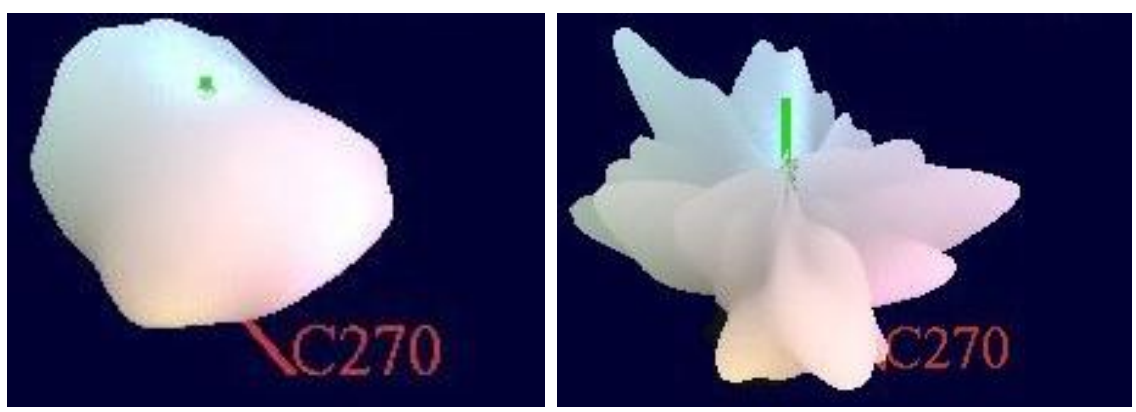

Figure 5: Spherical representation of an efficiency model taking different gloss value for optic and louvre as entries (normal to luminaire is upward). Left: optic gloss and louvre gloss are set to 0.5 and the efficiency is coherent with efficiencies in table 1 . Right: optic gloss is set to 0.5 while louvre gloss is set to 1 , leading to incoherent efficiency.

\subsection{Available model data}

The proposed $\mu\left(\begin{array}{l}C \\ \gamma\end{array}\right)$ model was constructed over the data obtained during efficiency extraction stages and exposed in table 1 . As expressed in equation 3 this leads to multiple linear regression parameters $\hat{A}$ every $3^{\circ}$ along $\mathrm{C} \in\left[0^{\circ} ; 360^{\circ}\right]$ and $\gamma \in\left[0^{\circ} ; 90^{\circ}\right]$. These 3,600 triplets are available online [17].

\subsection{Model accuracy}

The proposed $\mu\left(\begin{array}{l}C \\ \gamma\end{array}\right)$ method was evaluated on:

- the model accuracy relatively to experiment data

- the comparison between measured and computed irradiance.

\subsubsection{Model relative accuracy}

Lighting should be designed with quantity and quality objectives. On the one hand, irradiance (and illuminance) refers to light inflows. It mainly depends on the highest efficiency values for directions close to the normal to the luminaire (typically $\gamma<30^{\circ}$ ).

On the other hand, glare rating (e.g. UGR) refers to lighting quality and is mainly based on $\gamma>60^{\circ}$ emitting directions where efficiency values are the lowest.

That is why a relative accuracy metric was chosen, as defined in equation (6).

$$
\operatorname{Acc}\left(\begin{array}{l}
C \\
\gamma
\end{array}\right)=1-\left|\frac{\mu\left(\begin{array}{l}
C \\
\gamma
\end{array}\right)-\eta\left(\begin{array}{l}
C \\
\gamma
\end{array}\right)}{\eta\left(\begin{array}{l}
C \\
\gamma
\end{array}\right)}\right|
$$

In addition, we investigated whether the proposed method could be generalized from a subset to the whole available experiments. Apart from the available $\mu\left(\begin{array}{l}C \\ \gamma\end{array}\right)$ model data above, the 12 experiments were randomly distributed between a learning subset ( 8 experiments) and an appraisal subset (4 experiments). A new model was computed using the learning subset. Then its relative accuracy $A c c\left(\begin{array}{l}C \\ \gamma\end{array}\right)$ was computed relatively to the experiments

- of the learning subset (see table 2)

- of the appraisal subset (see table 3 ).

It appears that model accuracy will be the highest when computing irradiance and illuminance. On the contrary, UGR computed using the model may noticeably differ from the one computed using measured data.

In addition, there is no relationship between the "shape" of the efficiency and the accuracy of the model; e.g. experiments 3 and 5 from the learning subset have similar efficiency, whereas their accuracy differs notably. This is the same for experiments 9 and 10 from the learning subset, and experiments 7 and 8 from the appraisal subset. 
Table 2: Relative accuracy of the model computed from a "learning subset" of the available experiments: spherical representation of $\mu\left(\begin{array}{l}C \\ \gamma\end{array}\right)$ in false colors representing Acc $\left(\begin{array}{l}C \\ \gamma\end{array}\right)$, for experiments of the learning subset. Normal to emitting plane is upward.

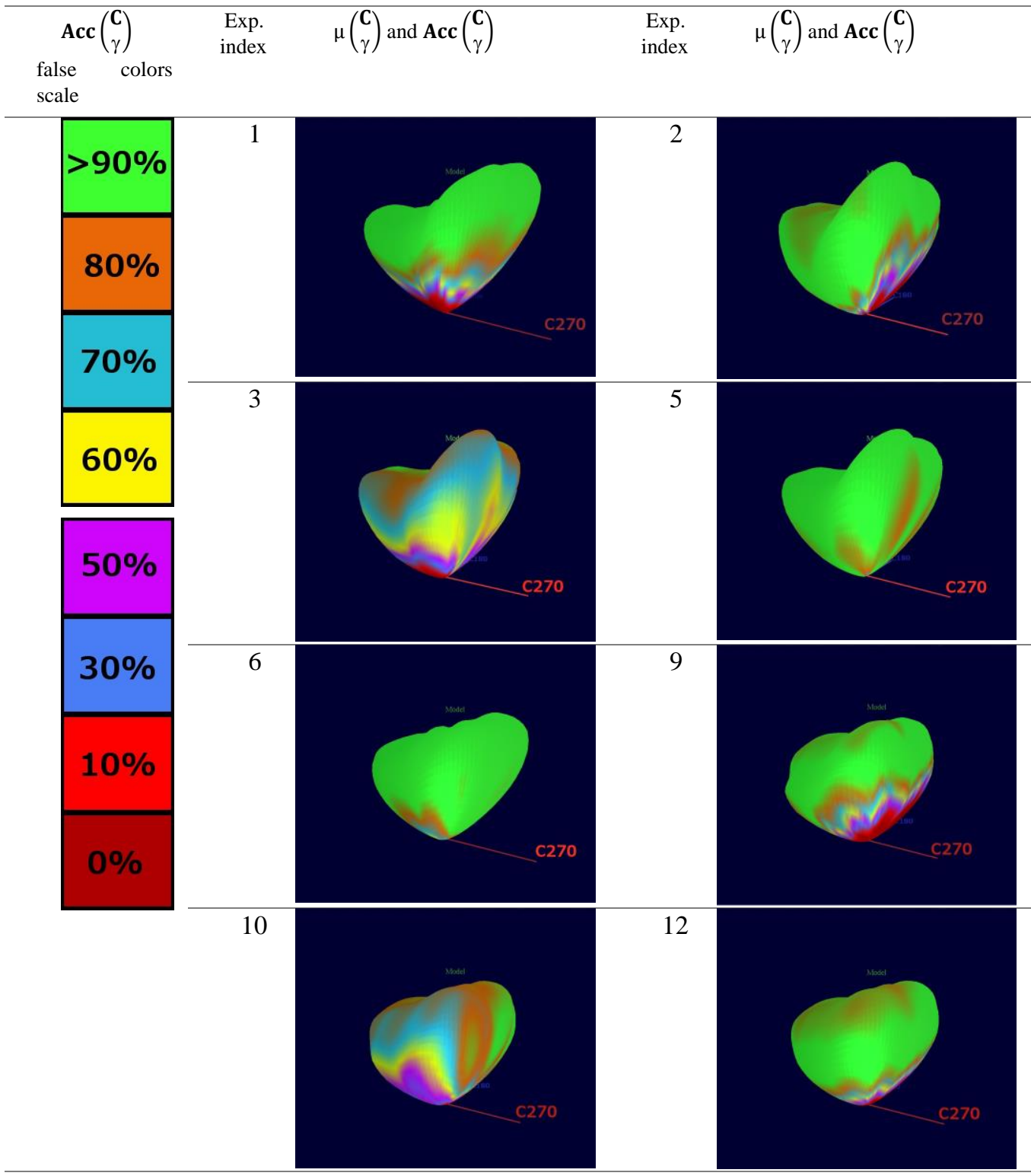


Table 3: Relative accuracy of the model computed from a "learning subset" of the available experiments: spherical representation of $\mu\left(\begin{array}{l}C \\ \gamma\end{array}\right)$ in false colours representing $A c c\left(\begin{array}{l}C \\ \gamma\end{array}\right)$, for experiments of the appraisal subset (i.e. not from the learning subset). Normal to emitting plane is upward.

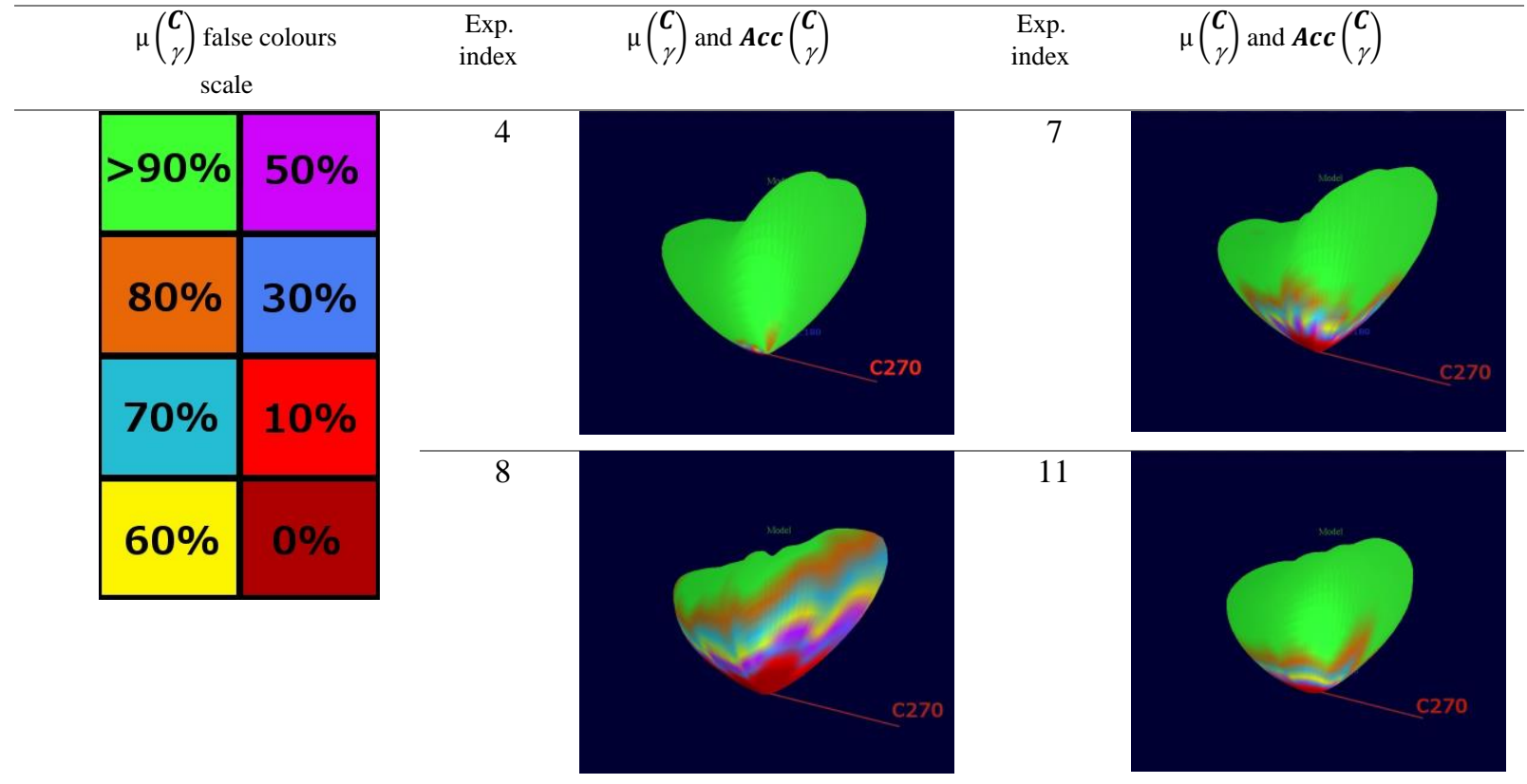

\subsubsection{Direct irradiance computation accuracy}

Comparisons were made, between:

- irradiance computed from Ee,OL750 $(\lambda)$ spectral irradiance measured with OL750 and

- irradiance computed using extracted efficiency and

- irradiance computed using modelled efficiency

in the $[380 \mathrm{~nm} ; 780 \mathrm{~nm}]$ range using the learning subset and the appraisal subset as described above.

The $0.6 \mathrm{~m} \times 0.6 \mathrm{~m}$ wide sample luminaires and the sensor faced each. Irradiance distance was $1.4 \mathrm{~m} \pm 5 \mathrm{~mm}$. In each case, irradiance distance was the same for measurement and simulation.

Simulations used the same lamps as during measurements. They consisted in four 18W Philips Master TLD Super 80 tubes.

As shown in tables 4 and 5, irradiance computed using extracted efficiency approached measurement in a $[-5 \% ;+19 \%]$ range.

Irradiance computed using modelled efficiency approached measurements within:

$-[-5 \% ;+11 \%]$ in the case of the learning subset (see table 4 ), and

$-[+10 \% ;+16 \%]$ in the case of the appraisal subset (see table 5 ).

These results indicate that the model is practically enough to approximate the learning data and other real luminaires. 
Table 4: Comparison between measured and computed irradiance, depending on the use of extracted or model efficiency. These experiments belong to the learning subset used to compute the efficiency model.

\begin{tabular}{|c|c|c|c|c|c|c|}
\hline \multirow[t]{2}{*}{ Exp. index } & \multicolumn{3}{|c|}{ Irradiance $\left(\mathrm{W} / \mathrm{m}^{2}\right)$} & \multicolumn{3}{|c|}{ Irradiance ratio } \\
\hline & Meas. & $\begin{array}{l}\text { Using } \\
\text { ex- } \\
\text { tracted } \\
\quad \text { data } \\
\end{array}$ & $\begin{array}{l}\text { Using } \\
\text { model }\end{array}$ & $\frac{\text { Extracted }}{\text { Measured }}$ & $\frac{\text { Model }}{\text { Measured }}$ & $\frac{\text { Model }}{\text { Extracted }}$ \\
\hline 1 & 2.2 & 2.3 & 2.3 & $105 \%$ & $105 \%$ & $100 \%$ \\
\hline 2 & 2.1 & 2.3 & 2.1 & $110 \%$ & $100 \%$ & $91 \%$ \\
\hline 3 & 1.9 & 1.9 & 2.1 & $100 \%$ & $111 \%$ & $111 \%$ \\
\hline 5 & 2.2 & 2.1 & 2.1 & $95 \%$ & $95 \%$ & $100 \%$ \\
\hline 6 & 2.1 & 2.3 & 2.2 & $109 \%$ & $105 \%$ & $96 \%$ \\
\hline 9 & 2.1 & 2.2 & 2.1 & $105 \%$ & $100 \%$ & $95 \%$ \\
\hline 10 & 1.9 & 1.8 & 2.1 & $95 \%$ & $111 \%$ & $117 \%$ \\
\hline 12 & 2.1 & 2.4 & 2.1 & $114 \%$ & $100 \%$ & $88 \%$ \\
\hline
\end{tabular}

\subsection{Model behavior when interpolating and extrapolating luminaire descriptors}

The goal of the proposed efficiency model is to produce the efficiency corresponding to a given luminaire description, in terms of optics width, optics gloss and louvre between-axis.

This model was constructed over and tested with a limited number of experiments and their corresponding descriptors. Its accuracy was appraised, compared to its learning subset of samples. As this was not enough to be confident in such a model, it was compared too to another appraisal subset of different samples. This comparison shows how the model fits to luminaires outside the learning subset.

Table 5: Comparison between measured and computed irradiance, depending on the use of extracted or modelled efficiency. These experiments belong to the appraisal subset, i.e. they were not used to compute the efficiency model.

\begin{tabular}{cccccccc}
\hline $\begin{array}{c}\text { Exp. } \\
\text { index }\end{array}$ & Dist. $(\mathrm{m})$ & \multicolumn{3}{c}{ Irradiance $\left(\mathrm{W} / \mathrm{m}^{2}\right)$} & \multicolumn{3}{c}{ Irradiance ratio } \\
\hline & & Meas. & $\begin{array}{c}\text { Using } \\
\text { ex- } \\
\text { tracted } \\
\text { data }\end{array}$ & $\begin{array}{c}\text { Using } \\
\text { model }\end{array}$ & $\frac{\text { Extracted }}{\text { Measured }}$ & $\frac{\text { Model }}{\text { Measured }}$ & $\frac{\text { Model }}{\text { Extracted }}$ \\
\hline $\mathbf{4}$ & 1.400 & 1.9 & 2.1 & 2.2 & $111 \%$ & $116 \%$ & $105 \%$ \\
\hline $\mathbf{7}$ & 1.410 & 2.1 & 2.5 & 2.3 & $119 \%$ & $110 \%$ & $92 \%$ \\
\hline $\mathbf{8}$ & 1.400 & 2.0 & 2.2 & 2.3 & $110 \%$ & $115 \%$ & $105 \%$ \\
\hline $\mathbf{1 1}$ & 1.398 & 1.9 & 2.1 & 2.2 & $111 \%$ & $116 \%$ & $105 \%$ \\
\hline
\end{tabular}

Knowing the behaviour of the model for particular samples was still not enough. It was necessary to ensure that the model results in coherent efficiencies whatever the descriptors values.

Table 6 gives an example of model efficiencies, as luminaire description evolves from one sample of the learning set to another. When descriptors are bounded by those of two sample luminaires of the learning set, they ware said interpolated. When at least one descriptor was not bounded, the descriptors configuration was said to be extrapolated.

Each row in the table corresponds to a change in one descriptor, whereas optics gloss or $\frac{\text { optic width }}{\text { louvre between-axis }}$. It is indicated whether the change corresponds to an interpolation or an extrapolation. 
Table 6: Coherence of model efficiencies as descriptors evolve from one sample to those of another. Changing descriptors are highlighted.

\begin{tabular}{|c|c|c|c|}
\hline Case & Gloss & $\frac{\text { optic width }}{\text { louvre between_axis }}$ & $\begin{array}{c}\text { Spherical representation } \\
\text { of } \mu\left(\begin{array}{l}\mathbf{C} \\
\gamma\end{array}\right)\end{array}$ \\
\hline $\begin{array}{l}\text { Modelled sample } \\
2\end{array}$ & 1 & 0.25 & \\
\hline Interpolation & & 0.25 & \\
\hline Interpolation & 0.7 & & \\
\hline Interpolation & 0.7 & & \\
\hline Extrapolation & 0.7 & & \\
\hline Extrapolation & & 4.0 & \\
\hline $\begin{array}{l}\text { Modelled sample } \\
1\end{array}$ & 1.0 & & \\
\hline
\end{tabular}

\section{Discussion}

An efficiency model for grid and tube luminaires with louvres is proposed. It was constructed over 12 experiments for which optic gloss and louvre between-axis vary.

As a first result, this work demonstrates that grid and louver spatial efficiency can be modelled precisely enough for lighting simulation, without requiring complex simulations. In addition, when constructed on a subset of these experiments, the model is able to predict the efficiency of the other experiments, accurately enough to cover real cases for the stated purpose. 
The proposed model accuracy and genericity are high enough so that the model can be integrated in lighting simulation software. This will help lighting designers to deal with existing lighting systems in case their photometry is not available.

A second result is this work corroborates the existence of empirical rules known by experienced lighting designers and manufacturers, in case they have to choose or conceive a reflector fitting at best a lighting situation. For each emitting direction, the efficiency model is made of a three components vector: a constant, an optics proportions multiplier and a gloss multiplier. At the difference of neural networks [9], these direction dependent vectors could be analysed to appraise the influence of each of these optics characteristics.

It should be noted that near-field spatial efficiency data were necessary to compute the proposed model. Data acquisition needs uncommon and expensive photometry devices (spectrophotometer and near-field goniophotometer) and noticeable filtering stages. Then spatial efficiencies are extracted from a several gigabytes large data set. The experimental setup as well as all these processes are complicated and not affordable for every lighting laboratory.

The proposed efficiency model shows several limitations addressed below.

\subsection{Sample set}

It may be argued that such a model should be obtained from a higher number of samples. First, available luminaires do not differ much in terms of optic width, louvre between axis and gloss. Second, unmounting louvres makes

$\frac{\text { optic width }}{\text { louvre between-axis }}$ range cover all non-exceptional cases.

It must be noted that a 13th sample was removed from the learning set because it showed an uncommon efficiency although it did not look special. This means it can exist some luminaires with which the proposed model does not fit.

At last, irradiance ratios in tables 4 and 5 show that the proposed method can be generalized from an even smaller learning subset. Generalization ability may be thoroughly studied by systematic n-fold cross-validation.

\subsection{Raw data filtering}

Raw photometric data were smoothed in order to construct the proposed model. This was necessary to avoid the model to introduce lighting uniformity anomalies in computations.

Examining $\frac{\text { Extracted }}{\text { Measured }}$ irradiance ratio exposed in tables 4 and 5, applied filters do not seem to induce noticeable inaccuracy, compared to other sources of inaccuracy while measuring as well as computing irradiance.

\subsection{Gloss}

Optic gloss has to be visually appraised by the model user. It ranges from 0 (matt) to 1 (mirror polish). As shown in table 6 , a 0.3 change results in a noticeably different efficiency.

It may be argued that such an effect on efficiency should require a precise gloss measurement. This is not feasible in practice because it would require unmounting an optic or louvre, then making it perfectly plane in order to use a glossmeter.

That is why:

- a software using this model should provide visual charts with reference gloss values,

- when analysing results from this model, limits on gloss accuracy should be taken into account.

\subsection{LED based luminaires}

It must be noted that this model is not intended at predicting the photometry of LED luminaires. Indeed, LED technology makes generally use of lenses and filters rather than reflector optics.

LED are small elements and cannot be modelled as lighting surfaces. They are point sources, which efficiency cannot be extracted the way exposed in this paper.

Usually LED light passes through an optical lens or sheet. All these are sophisticated and cannot be modelled simply, such as grids and louvers.

Even in the case of LED with reflectors, the current model cannot apply as the proportion of fluorescent tubes size compared to the optics is not that of LED.

For the future, this work opens several research directions. 
This work is an opening step in the efficiency-from-description field. Other light sources whose photometry is not always available, are to be taken into account. First, such sources are parabolic reflectors in which the primary light source (e.g. halogen filaments or arc lamps) is longitudinally aligned with the reflector. Second, parabolic projectors should be modelled, in which the reflector is a surface of revolution and the primary light source is axially positioned.

In addition, illumination from the proposed model is expressed as a linear combination of reflector characteristics once lamp flux and geometric factor are fixed. Search for optimal luminaire characteristics in order to obtain certain illuminance can take advantage of this relationship.

A simplistic example would be a set of i positions in space (i.e. a working plane) to be illuminated at a certain level $\mathrm{E}$, by a set of $\mathrm{j}$ louver luminaires of type $\left(\mathrm{P}=\frac{\text { optic width }}{\text { louvre between-axis }}, \mathrm{G}=\right.$ gloss $)$. All luminaires share the same $\varphi$ luminous power. Their position is fixed so that each $\Omega i j$ geometry term of $i$ illuminance from $j$ luminaire is determined.

Let's note $\widehat{A(\vec{\jmath})})$ the model value in the $\vec{\jmath} \mathbf{l}$ direction from luminaire $\mathrm{j}$ toward point $\mathrm{i}$. Finding optimal $\mathrm{P}$ and $\mathrm{G}$ would be minorizing $\Delta \mathrm{i}$ error in the following linear system :

$$
\forall i E=\varphi *(1 P G) . \sum_{j} \widehat{A(\vec{\jmath})} * \Omega_{i j}+\Delta_{i}
$$

As only $\mathrm{P}$ and $\mathrm{G}$ are unknown, an optimal $(\mathrm{P}, \mathrm{G})$ solution can be found using the same technic as for determining the luminaire model with equations (4) and (5).

\section{Conclusion}

Indoor lighting is often based on grid luminaires with tubes and louvres. These luminaires optic and louvre material and geometry are designed in order to provide a particular efficiency.

An efficiency model is proposed that fits the description of this kind of luminaires to its efficiency spatial distribution. It is especially useful when computing illuminance from unknown photometry luminaires - already installed luminaires to relamp or retrofit with LED tubes and unpublished photometry datasets.

The proposed method expresses efficiency values as the result of a multiple linear regression of the luminaire descriptors - ratio of optic width to louvre between-axis and gloss. For numerous emitting directions, multiple regression parameters are computed from a set of sample luminaires.

The proposed model enables reasonably accurate illuminance computation, though optic and louvres gloss are considered identical. Caution should be taken when rating glare. At last, this model is a first step toward efficiencyfrom-description: round and parabolic projectors are commonly used and should be modelled too.

In addition, such a linear model may be useful to solve the inverse problem, which is automatically suggesting lamp wattage and luminaires to achieve certain lighting quantity and quality, or suggesting reflector characteristics to obtain certain illuminance.

\section{References}

[1] Relux. (n.d.). Retrieved September 19, 2020, from https://reluxnet.relux.com/en/

[2] DIAL DIALux. (n.d.). Retrieved September 19, 2020, from https://www.dial.de/en/dialux/

[3] Cohen, M. F., \& Wallace, J. R. (2012). Radiosity and realistic image synthesis. Elsevier.

[4] David L.. DiLaura, Houser, K., Mistrick, R., \& Gary R.. Steffy. (2011). The Lighting Handbook: Reference and Application. Illuminating Engineering Society of North America.

[5] Baker, G. A., Heinisch, R. V., \& Lewin, I. (1977). New techniques for reflector design and photometry. Journal of the Illuminating Engineering Society, 6(4), 246-254.

[6] Synopsys LucidShape CAA V5 Based. (n.d.). Retrieved September 19, 2020, from https://www.synopsys.com/optical-solutions/lucidshape/caa-v5-based.html.

[7] Delacour, J. F., \& Cuinier, J. L. (2004, February). Presentation of the first PLM integrated Optical Simulation Software for the Design and Engineering of Optical Systems. In Optical Design and Engineering (Vol. 5249, pp. 42-53). International Society for Optics and Photonics.

[8] Krzysztof Wandachowicz (2012, September). Reflector calculation, validation, and optimization using Radiance. Presented at 11th International Radiance Workshop, Copenhagen.

[9] Delepoulle, S., Renaud, C., \& Preux, P. Photometric Compression and Interpolation for Light Source 
Representation.

[10] Delepoulle, S., Renaud, C., \& Preux, P. (2009). Light source storage and interpolation for global illumination: a neural solution. In Intelligent Computer Graphics 2009 (pp. 87-104). Springer, Berlin, Heidelberg.

[11] Chu, W., \& DiLaura, D. L. (1995). Improved Near-Field Illuminance Calculations Using Far-Field Photometry and Lum inance Scans. Journal of the Illuminating Engineering Society, 24(2), 3-7.

[12] de l'Éclairage, C. I. (1996). The photometry and goniophotometry of luminaires. Central Bureau of the CIE, Vienna,(121).

[13] Ashdown, I. (1993). Near-field photometry: a new approach. Journal of the Illuminating Engineering Society, 22(1), 163-180.

[14] Deniel, J. M. (2016). Optimizing array spectroradiometer readings using adaptative bracketing. Review of Scientific Instruments, 87(3), 033108.

[15] Carre, S. (1998). Architectural design tool for natural / artificial lighting. Application of image synthesis to take into account the concepts of visual comfort and ergonomics (Doctoral dissertation, Rennes 1).

[16] Deniel, JM (2002). Modeling of lights and BRDFs: realization, measurement and compression (Doctoral dissertation, Rennes 1).

[17] OSFhome Grid and Louvers photometry modeling (2018). Retrieved September 19, 2020, from https://osf.io/av bnw/ 